\title{
A UTILIZAÇÃO DE JOGOS E SIMULAÇÕES DE EMPRESAS NOS CURSOS DE GRADUAÇÃO EM ADMINISTRAÇÃO NO ESTADO DA BAHIA
}

\author{
Gustavo da Silva Motta \\ gustavosmotta@gmail.com \\ Universidade Federal Fluminense - Volta Redonda, RJ / Brasil \\ Rogério Hermida Quintella \\ rhquintella@gmail.com \\ Universidade Federal da Bahia - Salvador, BA / Brasil
}

Recebido em 31/05/2010

Aprovado em 02/06/2011

Disponibilizado em 01/08/2012

Avaliado pelo sistema double blind review

Revista Eletrônica de Administração

Editor: Luís Felipe Nascimento

ISSN 1413-2311 (versão on-line)

Editada pela Escola de Administração da Universidade Federal do Rio Grande do Sul.

Periodicidade: Quadrimestral

Sistema requerido: Adobe Acrobat Reader.

\section{RESUMO}

Este artigo apresenta um panorama sobre a utilização dos jogos de empresas na graduação em Administração pelas Instituições de Ensino Superior (IES) do estado da Bahia. Observa-se um crescente interesse de professores, no País e no Estado, na aplicação de jogos para a formação profissional do administrador. $\mathrm{O}$ aumento do interesse por essa técnica pode ser decorrente, entre outras origens, dos muitos benefícios que a literatura demonstra para seus usuários. Nesse artigo é desenvolvida uma revisão teórica dessa literatura buscando os seus conceitos centrais, a origem dos jogos e sua utilização no Brasil e no mundo. Em um segundo momento, apresenta-se dados de um levantamento realizado com 47 coordenadores de cursos de Administração de IES baianas sobre suas experiências institucionais com o uso da técnica. Optou-se por uma abordagem quantitativa com a utilização de estatísticas descritivas (frequência, média e desvio-padrão), constatando que 44,7\% das IES da Bahia aplicam o jogo para a formação de administradores. Igualmente, pode-se verificar que esta utilização deve crescer nos próximos cinco anos para $63,8 \%$, por causa dos elevados índices de satisfação com a aplicação e o mais alto grau de concordância com os aspectos favorecedores do que com os dificultadores à adoção do jogo. Finalmente, dois pontos se destacam como críticos a uma aplicação mais ampla dos jogos de empresas nos cursos de Administração no Estado da Bahia: o custo de aquisição e a oferta de simuladores eletrônicos.

Palavras-chave: Jogos de Empresas, Instituições de Ensino Superior (IES), Estado da Bahia. 


\title{
THE USE OF BUSINESS GAME AND SIMULATION IN UNDERGRADUATE BUSINESS PROGRAMS IN BAHIA STATE
}

\begin{abstract}
This paper presents an overview about the use of business simulation and games in undergraduate business programs in Bahia State, Brazil. The increasing interest about this technique can be originated, among others facts, on the many benefits that the specialized literature shows for its users. In this article a theoretical review on this literature is developed searching its central concepts, its origins and its use in Brazil and in the world. Some empirical data are presented from a survey with 47 under-graduation coordinators and directors from Bahian Institutions about their experience with business simulation and games by their lectures and professors. A quantitative approach using descriptive statistics (frequency, means and standard deviation) showed that $44.7 \%$ of the Bahian Institutions apply this kind of techniques. It shows also that business simulation practices are expected to grow in next five years, for $63,8 \%$ due to high satisfaction indications on its application. Finally, two aspects appeared as very important ones to a higher level of business simulation and games application in Management courses in Bahia: the acquisition cost and the availability of electronic simulations.
\end{abstract}

Keywords: Business Games, Higher Education Institutions, Bahia State.

\section{Introdução}

Os jogos de empresas começaram a ser utilizados na formação de administradores a partir da década de 1950 nos EUA. Desde então o número de professores, pesquisadores, consultores e profissionais que lidam com jogos e simulações vêm crescendo em diversos países, como destacado por Faria et al. (2009). No contexto brasileiro, esta utilização se deu a partir da década de 1970, tendo se intensificado somente a partir dos anos 2000, como destacado por Bernard (2006), Arbex et al. (2006), Paixão, Bruni e Carvalho (2007) e Neves e Lopes (2008).

Segundo Bernard (2006), dois são os principais motivos para a ampliação do número de aplicações de jogos de empresas: o surgimento de novos cursos de graduação em Administração, que de acordo com dados no INEP (2006) representam 16,4\% do total de alunos matriculados nos cursos de graduação presenciais do país; e a necessidade de adaptação às novas diretrizes curriculares do Ministério da Educação, para a qual o jogo de empresas é uma das formas alternativas de aprendizado propostas.

REAd I Porto Alegre - Edição 72 - N 2 - maio/agosto 2012 - p. 317-338 
Uma breve revisão da literatura (TANABE, 1973; KEYS e WOLFE, 1990; SAUAIA, 1995; LOPES, 2001; SHEIZI e SANTOS, 2003; OLIVIER e ROSAS, 2004; NAGAMATSU et al., 2006; LACRUZ e VILLELA, 2006; ROSAS e SAUAIA, 2006; BEN-ZVI, 2010) também aponta para os benefícios da técnica no desenvolvimento de diversas habilidades gerenciais na formação de administradores, tais como capacidade para tomada de decisões, reconhecimento do ambiente empresarial, comunicação interpessoal, dentre outras. Outro aspecto estimulador do uso dos jogos é a ubiquidade da microinformática que atualmente facilita a utilização de jogos e simuladores eletrônicos e permite uma maior complexidade às aplicações (FARIA, 1998; MENDES, 2000).

Ainda, ao observar a centralidade do papel dos alunos no processo de ensinoaprendizagem, quando submetidos à técnica de jogos de empresas, é possível contrastar suas características e resultados com outros métodos tradicionais que enfocam muito mais o papel do professor (SAUAIA, 1995), tais como as aulas expositivas. Há quase três décadas, Zajdsznajder (1981) observou a insatisfação dos alunos em relação à quantidade de conteúdo percebido como irrelevante à sua prática profissional. Certamente esta observação tem forte vocação utilitarista que coaduna com a visão de Bennis e O’Toole (2005). Para esses autores, “a administração é uma profissão, assim como a medicina e o direito, e escolas de gestão são escolas profissionais - ou deveriam ser" (BENNIS e O'TOOLE, 2005, p. 62). É importante deixar claro que há opiniões diversas a respeito desta ótica, que costumam ser enquadradas em um grande grupo, criando assim uma oposição. Este debate ficou conhecido como Relevância e Rigor (TUSHMAN, 2007). Independente do foco (na relevância profissional ou no rigor acadêmico) é comum, às diversas opiniões neste debate, a crítica ao processo de formação em administração e a visão de que o ensino nesses cursos deve buscar a relação com a prática da profissão de administrador (POLZER, et al, 2009; BERGGREN e SÖDERLUND, 2011). Desta forma, os jogos parecem apontar como uma das possibilidades de aplicação de recursos para o desenvolvimento das habilidades necessárias ao futuro administrador.

Por outro lado, mesmo com a crescente utilização dos jogos pelas Instituições de Ensino Superior (IES) brasileiras, a aplicação é relativamente pequena quando comparada a outros países, como Alemanha (PROTIL e FISCHER, 2003) e principalmente EUA. Faria (1998) apresenta dados sobre as instituições americanas que demonstram ampla utilização de jogos e simuladores, desde a década de 1960 (71,1\%), e um crescimento da aplicação que chegou a 97,5\% das 381 IES estudadas por ele em 1998. No Brasil, Bernard (2006) constatou

REAd I Porto Alegre - Edição 72 - Nº 2 - maio/agosto 2012 - p. 317-338 
que 61,4\% das IES aplicavam jogos de empresas para a formação de administradores em 2005. No mesmo período, Arbex et al. (2006) identificaram que, no Estado do Paraná, este índice era de cerca de $41 \%$ e Neves e Lopes (2008), no Estado de São Paulo, identificaram que $48,5 \%$ das IES locais utilizavam os jogos na graduação em administração. Apesar de se ter encontrado essas e outras informações sobre o uso da técnica em outros estados e países, não foram encontrados dados anteriores sobre a utilização de jogos na Bahia.

É nesse contexto que o presente artigo se propõe a apresentar os resultados de uma pesquisa, cujo objetivo foi a construção de um panorama sobre a utilização dos jogos de empresas na graduação em Administração pelas IES do Estado da Bahia. Tal proposta está apresentada em quatro partes, além desta introdução. Inicialmente é desenvolvida uma revisão teórica sobre o conceito e a origem dos jogos e sua utilização no Brasil e no mundo. Na sequência são apresentados os procedimentos metodológicos e os dados coletados na pesquisa. Finalmente, os dados são analisados e são apresentadas as considerações finais.

\section{Jogos de Empresas: origem e conceitos}

Inicialmente é importante estabelecer a diferenciação entre os conceitos de simulador e de jogo de empresas. Para Sauaia (2006) o simulador é um instrumento utilizado para a aplicação do jogo. Já o jogo em si é a dinâmica que simula (através de um software, ou não) as atividades de uma organização, onde os participantes vivenciam o processo de tomada de decisões. Assim, o simulador tem sua aplicação limitada à operacionalização de variáveis e a complexidade fica atribuída a partir da dinâmica do jogo com o ambiente de incerteza característico das decisões dos adversários e do comportamento do grupo no processo de negociação de uma decisão única.

Os jogos de empresas são oriundos dos jogos de guerra (TANABE, 1973; MARTINELLI, 1987; KEYS E WOLFE,1990), entretanto há divergências quanto ao local e época do aparecimento dos jogos. De acordo com Keys e Wolfe (1990), sua origem remonta a 3.000 anos a.C, com a simulação de guerra Wei-Hái, na China, e com o jogo Chaturanga, na Índia. Por outro lado, Tanabe (1973) e Martinelli (1987) afirmam que os jogos surgiram no século XIX desenvolvidos inicialmente pelo exército prussiano. Segundo Keys e Wolfe (1990), o mais elaborado dentre eles, o New Kriegspiel, teria sido criado por George Venturini, em Schleswig, já em 1798. Tais jogos de guerra evoluíram para versões de jogos REAd I Porto Alegre - Edição 72 - N 2 - maio/agosto 2012 - p. 317-338 
empresariais. Segundo Faria (1998), primeiro simulador desenvolvido para a aplicação de jogos de empresas foi o Monopologs da Rand Corporation criado em 1955. O Monopologs focava o sistema logístico da Força Aérea Americana. Em 1956, a American Management Association (AMA) desenvolveu aquele que viria a ser o primeiro simulador amplamente conhecido, o Top Management Decision Simulation.

A primeira utilização dos jogos em IES, segundo Faria (1998) e Keys e Wolfe (1990), data de 1957, quando o Top Management Decision Game desenvolvido por Schreiber, que teve como modelo o Top Management Decision Simulation, foi levado para a Universidade de Washington a fim de ser incorporado ao método de ensino empregado em sala de aula. No Brasil, os jogos de empresas foram utilizados, como técnica de ensino nos cursos universitários, a partir da década de 1970, como relatado por Goldschmidt (1977), numa das primeiras experiências brasileiras, ocorrida na EAESP (Escola de Administração de Empresas de São Paulo).

Na visão de Goldschmidt (1977), a utilização desse instrumento no Brasil começou praticamente da mesma forma em todas as IES: pela importação de jogos americanos na década de 70. Com o decorrer do tempo, aliando-se o feedback dos participantes ao aprendizado dos instrutores, deu-se início ao desenvolvimento dos jogos de empresas nacionais.

Com o progresso da tecnologia de microinformática, tanto do ponto de vista de hardware como de software, os jogos de empresa migraram dos mainframes para os microcomputadores permitindo a elaboração de modelos mais fáceis de operar, dinâmicos e velozes (FARIA, 1998; MENDES, 2000). Com o desenvolvimento de novas técnicas pedagógicas, baseadas na exploração de vivências dos participantes, os jogos de empresa tiveram um grande impulso como instrumento de treinamento e desenvolvimento gerencial (WILHELM E LOPES, 1997).

Diversos autores conceituam jogos de empresas com diferentes visões em épocas diferentes. Embora possam divergir em termos de abrangência e profundidade, todos concordam no que diz respeito à dinâmica essencial e seus pressupostos pedagógicos. Keys e Wolfe (1990), dois dos mais importantes estudiosos internacionais sobre a temática, definem jogos de empresas como sendo:

REAd I Porto Alegre - Edição 72 - N 2 - maio/agosto 2012 - p. 317-338 
uma situação experimental, na qual aprendizagem e mudanças de comportamento podem ocorrer, e na qual comportamentos de gestão possam ser observados. Uma simulação experimental é uma situação simplificada que contém suficiente semelhança, ou ilusão da realidade, para induzir respostas nos exercícios por parte dos participantes de forma semelhante à vida real (KEYS e WOLFE, 1990, p. 1)

Sauaia (1995), um dos mais expressivos pesquisadores brasileiros na área, define jogo de empresas como "um método de aprendizagem que permite aos participantes o desempenho de papéis gerenciais em empresas fictícias" (SAUAIA, 1995, p. 9).

Rocha (1997) descreve jogo de empresas como sendo uma simulação do ambiente empresarial, tanto em seus aspectos internos como externos, que permitem a avaliação e a análise das possíveis conseqüências decorrentes de decisões abordadas. Mais recentemente, Santos (2003) elaborou a seguinte definição:

Os jogos de empresas são abstrações matemáticas simplificadas de uma situação relacionada com o mundo dos negócios. Os participantes administram a empresa como um todo ou em parte dela, através de decisões seqüenciais. Os jogos de empresas também podem ser definidos como um exercício de tomada de decisões em torno de um modelo de operação de negócios, no qual os participantes assumem o papel de administradores de uma empresa simulada podendo assumir diversos papéis gerenciais, funcionais, especialistas e generalistas. (SANTOS, 2003, p. 83)

Percebe-se que, em sua maioria, as definições apresentadas coincidem no argumento de que jogos de empresa são modelos dinâmicos de simulação que salientam as situações da área empresarial, bem como o treinamento para o processo de tomada de decisões. Pode-se observar que jogos de empresa representam uma técnica educacional dinâmica, desenvolvida para propiciar aos jogadores/aprendizes uma experiência de aprendizagem marcante e lúdica. Assim, os jogos funcionam como uma ponte entre a academia e o ambiente empresarial, a partir de uma representação da realidade (situações específicas da área empresarial) por meio de abstrações matemáticas que se utilizam de técnicas de simulação (retratando condições de laboratório de uma determinada realidade não sendo somente uma simulação da empresa, mas do mercado) e possuem componentes dos jogos (trazendo a interatividade e o exercício em equipe).

Todas as definições apresentam a relação dos jogos com conceitos e pressupostos mais amplos e clássicos, tais como o da aprendizagem vivencial (KOLB, 1984). Segundo Kolb (1984), a aprendizagem vivencial considera o aprendizado como um processo, no qual o conhecimento é produzido por meio da transformação da experiência. Dessa forma, é possível extrair três aspectos fundamentais da aprendizagem vivencial. Inicialmente, observa-se uma 
ênfase no processo e na aprendizagem, em oposição à ênfase no conteúdo e no resultado, característicos do ensino tradicional. Segundo, o conhecimento é visto como um processo de transformação que é continuamente criado e recriado, ao invés de um produto acabado que deve ser adquirido ou transmitido. Finalmente, a aprendizagem vivencial reconhece que os indivíduos aprendem de diferentes formas e respondem distintamente a um mesmo estímulo (KOLB, 1984; THORELLI, 2001; PEREIRA, 2005).

\section{A Utilização de Jogos de Empresas no Brasil e no Mundo}

Faria (1987) desenvolveu uma pesquisa seminal sobre a intensidade e a abrangência do uso de Jogos de Empresas nos EUA. Este trabalho foi atualizado uma década depois (FARIA, 1998), demonstrando o crescimento da utilização de jogos neste período e inferindo uma expectativa de crescimento futuro naquele país.

Faria (1998) apresenta dados de pesquisas anteriores e os compara às suas, constatando que o uso de jogo de empresas para o ensino de administração nas IES tem crescido constantemente nos EUA desde a década de 1960, como apresentado na Tabela 1.

Tabela 1: Uso de Jogos nos EUA

\begin{tabular}{lc}
\hline Estudo & Utilização (\%) \\
\hline Dale e Klasson (1962) & 71,1 \\
Graham e Gray (1969) & 90,7 \\
Roberts e Strauss (1975) & 94,5 \\
Faria (1987) & 95,1 \\
Faria (1998) & 97,5 \\
\hline \multicolumn{2}{c}{ Fonte: Adaptado de Faria (1998) }
\end{tabular}

Outros pesquisadores também se preocuparam em publicar trabalhos de investigação sobre a adoção de jogos de empresas para a formação de administradores. Burgess (1991) apresenta o uso de jogos de empresas no Reino Unido, McKenna (1991) na Austrália, Chang (2003) em Hong Kong e Protil e Fischer (2003) na Alemanha. No Brasil, Bernard (2006) realizou um levantamento, em 2005, sobre a aplicação de jogos de empresas pelas IES em todo o país. No mesmo período, Arbex et al. (2006) investigaram a utilização no estado do Paraná e Neves e Lopes (2008), em 2007, no estado de São Paulo.

Estados Unidos, Inglaterra e Alemanha são alguns dos países que sediam as reuniões anuais de associações nacionais e internacionais. As internacionais ocorrem em diferentes 
países do mundo deslocando-se, a cada ano, através da Europa, América e Ásia. Destacam-se, entre elas, as seguintes associações (KEYS, 1990):

- ABSEL (Association For Business Simulation and Experiential Learning) - EUA;

- NASAGA (North American Simulation and Gaming Association) - EUA;

- OBTC (Organizational Behavior Teaching Society) - EUA;

- ISAGA (International Simulation and Gaming Association) - Inglaterra;

- SAGSET (Society For the Advancement of Games and Simulation in Education and Training) - Inglaterra;

- EPS (Europaisches Planspiel Forum) - Alemanha.

No Brasil, observa-se que duas IES têm notoriedade no papel de desenvolvedores, usuários e pesquisadores de jogos de empresas. A Universidade Federal de Santa Catarina (UFSC), em seu curso de pós-graduação stricto sensu em Engenharia da Produção e Sistemas, tem contribuído muito com o desenvolvimento de diversos jogos de empresa (GI-MICRO; GI-EPS; LIDERSIT; LIDER; GS-ENE; GEBAN; GI-LOG; dentre outros) e de dezenas de dissertações e teses, muitas das quais propõem adaptações em jogos já existentes ou criam novos jogos. Da mesma forma os cursos de pós-graduação stricto sensu da Faculdade de Economia, Administração e Contabilidade da Universidade de São Paulo (FEA/USP), de onde se originaram, no Brasil, as primeiras dissertações (TANABE, 1973; BEPPU, 1984; MARTINELLI, 1987; SAUAIA, 1990) e tese (SAUAIA, 1995) sobre jogos de empresa, contribuem para a evolução de pesquisas sobre o assunto.

Recentemente, têm-se desenvolvido, no Brasil, algumas disputas de simulação empresarial voltadas para graduandos via internet, com custos baixos para os participantes o que tem permitido a difusão da utilização de jogos de empresas no ensino superior, podendose citar os eventos: Torneio de Simulação Empresarial, promovido pela Bernard Sistemas; Desafio Sebrae, promovido pelo Sebrae; Torneio de Jogos de Empresas, promovido pela UFF (Universidade Federal Fluminense); Olimpíada Universitária, realizada pela MicrosigaIntelligence e a Copa Universitário, realizada pela Simulation e Associados, esta desde 1999 e as demais a partir de 2000 .

REAd I Porto Alegre - Edição 72 - N 2 - maio/agosto 2012 - p. 317-338 


\section{Aspectos Dificultadores e Favorecedores à Aplicação de Jogos de Empresas}

Neves e Lopes (2008) desenvolveram uma classificação dos aspectos que dificultam e daqueles que favorecem a adoção de jogos de empresas pelas IES. Baseando a descrição dos aspectos dificultadores na classificação de Goosen et al. (2001), Neves e Lopes (2008) afirmam que tais aspectos são, em sua maioria, evitáveis, ou seja, que podem ser controlados através da gestão. Segundo Goosen et al. (2001), a origem dos aspectos que dificultam a aplicação dos jogos pode estar relacionada aos participantes, aos desenvolvedores e aos aplicadores (que podem ser IES ou professores isolados) do jogo.

No caso específico dos aplicadores, Neves e Lopes (2008) identificaram, numa revisão de literatura, alguns pontos como os principais dificultadores da aplicação de jogos:

a) Falta de professores treinados para animar jogos de empresas (LOPES, 2001; ARBEX et al, 2006; ROSAS; SAUAIA, 2006);

b) Elevado custo de aquisição dos jogos (ARBEX et al, 2006; ROSAS; SAUAIA, 2006);

c) Dificuldade de acesso a jogos prontos (LOPES, 2001);

d) Infraestruturas inadequadas à aplicação de jogos nas IES (ARBEX et al, 2006; ROSAS; SAUAIA, 2006);

e) Falta de consciência de coordenadores e IES (ROSAS; SAUAIA, 2006).

Neves e Lopes (2008) adicionaram ainda, em sua pesquisa, aspectos relacionados à falta de verossimilhança dos jogos com o contexto empresarial brasileiro e a elevada demanda de tempo para aplicação da técnica.

No que tange os aspectos favoráveis à adoção de jogos de empresas nos cursos de graduação em administração, Rosas e Sauaia (2006) identificaram fatores que, numa perspectiva futura, incentivaria o uso de jogos. Estes fatores foram definidos por Neves e Lopes (2008) da seguinte forma:

a) Importância dos jogos na formação profissional do administrador;

b) O elevado interesse dos alunos pela dinâmica dos jogos;

c) Importância da aprendizagem cognitiva proporcionada pelos jogos;

d) Aproximação entre a teoria e a prática;

REAd I Porto Alegre - Edição 72 - N 2 - maio/agosto 2012 - p. 317-338 
e) O estímulo ao pensamento sistêmico;

f) O estímulo ao trabalho em equipe;

g) A integração dos conteúdos das diversas disciplinas do curso de administração.

Espera-se que o reconhecimento desses fatores seja favorável a uma aplicação mais ampla dos jogos de empresas para a formação de administradores. A partir do momento em que IES, representadas por seus coordenadores de curso e professores, percebem o valor de tal aplicação, a tendência é a utilização dos jogos nas diversas disciplinas específicas do curso de administração.

\section{Procedimentos Metodológicos}

Optou-se, nesta pesquisa, por uma abordagem que pode ser classificada como quantitativa e descritiva. Foram utilizados, como instrumento de coleta de dados, questionários de autoaplicação, contendo questões abertas e fechadas, que foram enviados por correio eletrônico e, em alguns casos, administrados presencialmente na ocasião do IV EPROCAD - Encontro Regional de Professores e Coordenadores de Cursos de Administração - realizado em 07 de novembro de 2008 na cidade de Salvador/BA. Os questionários foram enviados aos coordenadores de todas as 92 IES que oferecem o curso de Administração no Estado da Bahia, cadastradas no INEP (Instituto Nacional de Estudos e Pesquisas Educacionais Anísio Teixeira). Num segundo momento, aquelas IES que não haviam respondido ao questionário foram contatadas por telefone. No total, obteve-se 47 respostas. Para a coleta de dados, as 92 IES foram divididas em dois estratos: 43 da Região Metropolitana de Salvador (RMS) e 49 do Interior do estado. A Tabela 2 apresenta a relação entre a amostra e o universo por estrato.

Tabela 2: IES por estrato

\begin{tabular}{lccc}
\hline \multicolumn{1}{c}{ Estrato } & Universo & $\begin{array}{c}\text { Amostra } \\
\text { Absoluta }\end{array}$ & $\begin{array}{c}\text { Amostra } \\
\text { Relativa }\end{array}$ \\
\hline 1-RMS & 43 & 23 & $53,5 \%$ \\
2-Interior & 49 & 24 & $48,9 \%$ \\
\hline Total & $\mathbf{9 2}$ & $\mathbf{4 7}$ & $\mathbf{5 1 , 1 \%}$ \\
\hline \multicolumn{5}{c}{}
\end{tabular}


Observa-se que esta amostra (47) é representativa do seu universo (92), considerando um intervalo de confiança de $95 \%$ e erro amostral admitido de $10 \%$, de acordo com a técnica de cálculo amostral apresentada por Aaker, Kumar e Day (2004).

O instrumento de coleta de dados foi construído em duas partes. A primeira parte buscava investigar a utilização de jogos de empresas pelas IES baianas em dois níveis: como um componente curricular e/ou como atividade dentro de outras disciplinas. Assim, buscou-se verificar, dentre aquelas que ofereciam como componente curricular, quais os objetivos de aprendizagem da disciplina. Verificou-se também, independente do nível de utilização: i) o grau de satisfação institucional; ii) se fazia uso de software; iii) qual o software utilizado e quem o desenvolveu; iv) e qual o motivo para a escolha do software. Para aquelas que não utilizavam software, buscou-se investigar: i) como eram aplicados os jogos; ii) e quais os motivos para a escolha desta forma de aplicação. Para aquelas IES que não aplicavam o jogo, verificou-se: i) o motivo da não-utilização; ii) e se havia pretensão de utilização nos próximos cinco anos.

A segunda parte do questionário foi construída numa escala tipo Likert de cinco pontos, com respostas variando entre baixa (1) a alta concordância (5), conforme apresentado na Figura 1. Esta escala foi adaptada daquela utilizada e validada por Neves e Lopes (2008), que encontraram um resultado de 0,9649 para o coeficiente alpha de Cronbach.

Figura 1: Escala para aspectos favoráveis e desfavoráveis ao jogo de empresas

\begin{tabular}{|ccccc|}
\hline 1 & 2 & 3 & 4 & 5 \\
$\begin{array}{c}\text { Discordo } \\
\text { Totalmente }\end{array}$ & Discordo & Indiferente & Concordo & $\begin{array}{c}\text { Concordo } \\
\text { Totalmente }\end{array}$ \\
\hline
\end{tabular}

Fonte: Elaborado pelos autores com base em Neves e Lopes (2008)

Buscou-se, através desta escala identificar a avaliação dos coordenadores de curso das IES pesquisadas a respeito dos aspectos favoráveis e desfavoráveis à utilização dos jogos de empresas para a formação de administradores.

Os dados foram coletados entre os meses de novembro de 2008 e janeiro de 2009. Após a coleta, os dados foram tabulados em planilha eletrônica e analisados, estatisticamente, com o uso do software PASW Statistcs 17.0. A análise dos dados foi realizada através de estatísticas descritivas (freqüência, média e desvio-padrão).

\section{Análise dos Dados}

REAd I Porto Alegre - Edição 72 - N 2 - maio/agosto 2012 - p. 317-338 
Todos os 47 coordenadores de curso pesquisados afirmaram conhecer o jogo de empresas enquanto técnica de ensino. Sobre a utilização dos jogos, 40,4\% afirmaram utilizar em uma disciplina específica, denominada "Jogos de Empresas" e outros 34,1\% informaram utilizar em outras disciplinas, o que totaliza 74,5\% que declararam utilizar jogos de empresas para a formação de administradores. Entretanto, dessas IES, 54,3\% utilizam um software, enquanto as demais declararam desenvolver a técnica de formas variadas, como pode ser observado na Tabela 3. Percebe-se que muitos daqueles que afirmam aplicar jogos e não utilizam um software para tal, demonstram um equívoco em relação à técnica ao afirmarem que tal procedimento é feito através de: i) dinâmicas comportamentais (50\%); ii) casos para estudos (25\%); iii) e até declarações a respeito de improviso (12,5\%). Claramente, essas não são aplicações de jogos de empresas.

Tabela 3: Dinâmicas de aplicação sem software

\begin{tabular}{lccc}
\hline \multicolumn{1}{c}{ Dinâmica } & $\begin{array}{c}\text { Frequência } \\
\text { Absoluta }\end{array}$ & $\begin{array}{c}\text { Frequência } \\
\text { Relativa }\end{array}$ & $\begin{array}{c}\text { Frequência } \\
\text { Relativa } \\
\text { Acumulada }\end{array}$ \\
\hline Dinâmicas Comportamentais & 8 & $50,0 \%$ & $50,0 \%$ \\
Casos para estudo & 4 & $25,0 \%$ & $75,0 \%$ \\
Improviso & 2 & $12,5 \%$ & $87,5 \%$ \\
Jogos de sala de aula & 2 & $12,5 \%$ & $100,0 \%$ \\
\hline Total que utiliza & $\mathbf{1 6}$ & $\mathbf{1 0 0 \%}$ & - \\
\hline
\end{tabular}

Fonte: Elaborado pelos autores

Apenas 12,5\% aplicam, efetivamente, jogos sem a utilização de software. Desta forma, evidencia-se que o índice real de uso de jogo de empresas pelas IES baianas é de 44,7\%, dados similares aos encontrados por Arbex et al. (2006) para o estado do Paraná e Neves e Lopes (2008) para o estado de São Paulo, apresentados anteriormente. Observa-se ainda que, mesmo existindo o componente curricular "Jogos de Empresas" em 40,4\% das IES, apenas $23,4 \%$, ou seja, $57,9 \%$ delas aplicam jogo na formação de administradores e outras $21,3 \%$ aplicam o jogo dentro de outras disciplinas. A Tabela 4 apresenta os dados sobre a utilização, após o devido tratamento.

Tabela 4: Utilização de jogo de empresas pelas IES baianas

\begin{tabular}{lccc}
\hline \multicolumn{1}{c}{ Utilização } & $\begin{array}{c}\text { Frequência } \\
\text { Absoluta }\end{array}$ & $\begin{array}{c}\text { Frequência } \\
\text { Relativa }\end{array}$ & $\begin{array}{c}\text { Frequência } \\
\text { Relativa } \\
\text { Acumulada }\end{array}$ \\
\hline Sim, em disciplina específica & 11 & $23,4 \%$ & $23,4 \%$ \\
Sim, em outras disciplinas & 10 & $21,3 \%$ & $44,7 \%$ \\
\hline Total que utiliza & $\mathbf{2 1}$ & $\mathbf{4 4 , 7 \%}$ & - \\
\hline Não, mas pretende (já está trabalhando) & 3 & $6,4 \%$ & $51,1 \%$ \\
Não, mas pretende (não está trabalhando) & 6 & $12,7 \%$ & $63,8 \%$ \\
\hline Total que planeja utilizar & $\mathbf{9}$ & $\mathbf{1 9 , 1 \%}$ & - \\
\hline
\end{tabular}

REAd I Porto Alegre - Edição 72 - Nº 2 - maio/agosto 2012 - p. 317-338 


\begin{tabular}{lccc}
\hline Não e não planeja & 3 & $6,4 \%$ & $70,2 \%$ \\
\hline Não, mas afirma utilizar & 14 & $29,8 \%$ & $100,0 \%$ \\
\hline Total & $\mathbf{4 7}$ & $\mathbf{1 0 0 , 0 \%}$ & - \\
\hline
\end{tabular}

Fonte: Elaborado pelos autores

Igualmente pode-se verificar que a utilização de jogo de empresas pelas IES do Estado da Bahia deve crescer, nos próximos cinco anos, para 63,8\%, o que aproximaria este índice ao identificado por Bernard (2006) em seu levantamento nacional.

Com este tratamento dos dados, constata-se que a utilização do software é determinante na aplicação de jogo de empresas pelas IES baianas, como demonstrado na Figura 2.

Figura 2: Utilização de simulador eletrônico (software)

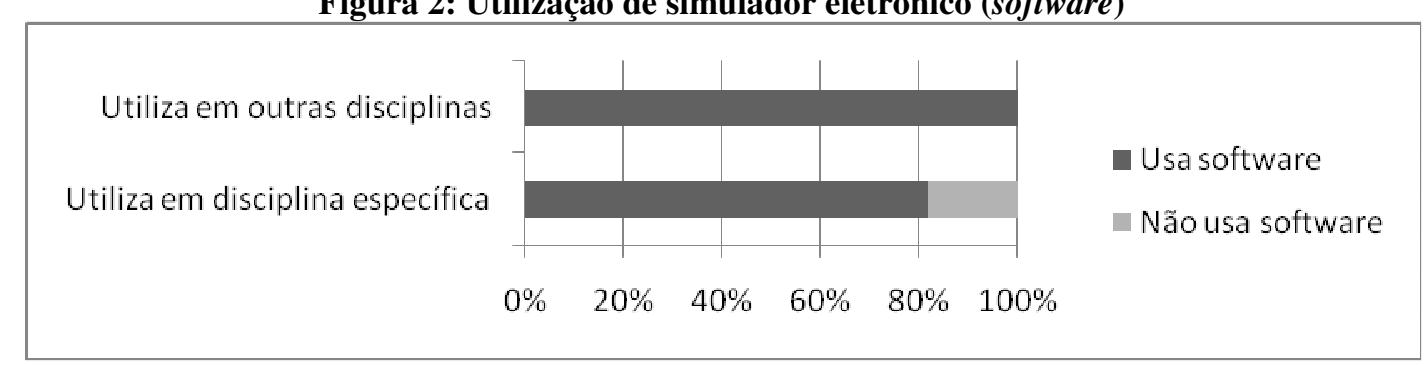

Fonte: Elaborado pelos autores

Observa-se ainda, a respeito do uso de software, que apenas dois deles (GI Micro desenvolvido pela UFSC e o Bernard da Bernard Sistemas) são responsáveis por, pelo menos 36,8\% das aplicações (Tabela 5). Ou seja, há uma concentração de poucos aplicativos e uma preferência pelos que são desenvolvidos nacionalmente.

Tabela 5: Softwares utilizados

\begin{tabular}{lccc}
\hline Software & $\begin{array}{c}\text { Frequência } \\
\text { Absoluta }\end{array}$ & $\begin{array}{c}\text { Frequência } \\
\text { Relativa }\end{array}$ & $\begin{array}{c}\text { Frequência } \\
\text { Relativa } \\
\text { Acumulada }\end{array}$ \\
\hline GI Micro (UFSC) & 5 & $26,3 \%$ & $26,3 \%$ \\
Bernard (Bernard Sistemas) & 2 & $10,5 \%$ & $36,8 \%$ \\
Outros & 6 & $31,6 \%$ & $68,4 \%$ \\
Não sabe & 6 & $31,6 \%$ & $100,0 \%$ \\
\hline Total & $\mathbf{1 9}$ & $\mathbf{1 0 0 , 0 \%}$ & - \\
\hline
\end{tabular}

Fonte: Elaborado pelos autores

Dentre as IES que aplicam jogos de empresas como uma disciplina, buscou-se verificar qual o principal objetivo de aprendizagem da mesma, obtendo-se o resultado conforme Tabela 6.

REAd I Porto Alegre - Edição 72 - Nº 2 - maio/agosto 2012 - p. 317-338 
A utilização de jogos e simulações de empresas nos cursos de graduação em

Administração no estado da Bahia

Tabela 6: Principal objetivo de aprendizagem da disciplina Jogos de Empresas

\begin{tabular}{lccc}
\hline \multicolumn{1}{c}{ Objetivo } & $\begin{array}{c}\text { Frequência } \\
\text { Absoluta }\end{array}$ & $\begin{array}{c}\text { Frequência } \\
\text { Relativa }\end{array}$ & $\begin{array}{c}\text { Frequência } \\
\text { Relativa } \\
\text { Acumulada }\end{array}$ \\
\hline Integrar os conteúdos das diversas disciplinas do curso & 8 & $42,1 \%$ & $42,1 \%$ \\
Oferecer vivência empresarial & 6 & $31,5 \%$ & $73,6 \%$ \\
Treinar para o processo de tomada de decisões & 2 & $10,5 \%$ & $84,1 \%$ \\
Aliar teoria e prática & 1 & $5,3 \%$ & $89,4 \%$ \\
Estimular o empreendedorismo & 1 & $5,3 \%$ & $94,7 \%$ \\
Desenvolver técnicas de negociação & 1 & $5,3 \%$ & $100,0 \%$ \\
\hline Total que utiliza & $\mathbf{1 9}$ & $\mathbf{1 0 0 , 0 \%}$ & - \\
\hline
\end{tabular}

Fonte: Elaborado pelos autores

Três objetivos correspondem a $84,1 \%$ daqueles declarados como os principais objetivos de aprendizagem da disciplina. É importante destacar que tais objetivos (integrar os conteúdos das diversas disciplinas do curso; oferecer vivência empresarial; e treinar para o processo de tomada de decisões) encontram elevada congruência com os conceitos e recomendações de diversos dos autores apresentados neste artigo.

Buscou-se então identificar quais os motivos que levavam as IES a escolher determinado software ou outra forma de aplicação do jogo. Foi constatado que 57,9\% das escolhas de um software específico são determinadas pelos custos e somente 26,3\% pelos objetivos de aprendizagem ou indicação do professor, como se vê na Tabela 7.

Tabela 7: Motivo da escolha do simulador

\begin{tabular}{lccc}
\hline \multicolumn{1}{c}{ Motivo } & $\begin{array}{c}\text { Frequência } \\
\text { Absoluta }\end{array}$ & $\begin{array}{c}\text { Frequência } \\
\text { Relativa }\end{array}$ & $\begin{array}{c}\text { Freq. Relativa } \\
\text { Acumulada }\end{array}$ \\
\hline Simulador eletrônico (software) & & & \\
$\quad$ Adequação aos objetivos da disciplina & 3 & $15,8 \%$ & $15,8 \%$ \\
$\quad$ Indicação do professor & 2 & $10,5 \%$ & $26,3 \%$ \\
Custo & 11 & $57,9 \%$ & $84,2 \%$ \\
$\quad$ Não sabe & 3 & $15,8 \%$ & $100,0 \%$ \\
\hline Total simulador eletrônico & $\mathbf{1 9}$ & $\mathbf{1 0 0 , 0 \%}$ & - \\
\hline Simulador Manual & & & \\
$\quad$ Ausência de software & 1 & $50,0 \%$ & $50,0 \%$ \\
$\quad$ Custo de software elevado & 1 & $50,0 \%$ & $100,0 \%$ \\
\hline Total Simulador Manual & $\mathbf{2}$ & $\mathbf{1 0 0 , 0 \%}$ & - \\
\hline
\end{tabular}

Fonte: Elaborado pelos autores

Quando a aplicação do jogo se dá sem a utilização de software, percebe-se que a opção pela aplicação manual é feita por conta do suposto "elevado custo de aquisição" de softwares. A importância desse custo também se destaca quando se observa os motivos da não-aplicação de jogo pelas IES, expostos na

Tabela 8.

REAd I Porto Alegre - Edição 72 - Nº 2 - maio/agosto 2012 - p. 317-338 
Tabela 8: Motivo da não aplicação de jogos

\begin{tabular}{lccc}
\hline \multicolumn{1}{c}{ Motivo } & $\begin{array}{c}\text { Frequência } \\
\text { Absoluta }\end{array}$ & $\begin{array}{c}\text { Frequência } \\
\text { Relativa }\end{array}$ & $\begin{array}{c}\text { Frequência } \\
\text { Relativa } \\
\text { Acumulada }\end{array}$ \\
\hline Custo de software elevado & 7 & $58,3 \%$ & $58,3 \%$ \\
Faltam professores capacitados & 2 & $16,7 \%$ & $75,0 \%$ \\
Ausência de software & 1 & $8,3 \%$ & $83,3 \%$ \\
Desenvolvem outra atividade com o mesmo efeito & 1 & $8,3 \%$ & $91,6 \%$ \\
Não sabe & 1 & $8,3 \%$ & $100,0 \%$ \\
\hline Total & $\mathbf{1 2}$ & $\mathbf{1 0 0 , 0 \%}$ & - \\
\hline
\end{tabular}

Fonte: Elaborado pelos autores

Numa escala de três pontos, identifica-se também um índice de satisfação institucional com a aplicação de jogos através do uso de software mais elevado $(2,74)$ do que de outras formas $(2,44)$. Por outro lado, a existência de uma disciplina específica não influencia o índice de satisfação. A Tabela 9 apresenta as médias e desvios padrão de acordo com as formas de aplicação dos jogos.

Tabela 9: Satisfação com a aplicação de Jogos

\begin{tabular}{cccc}
\hline \multicolumn{4}{c}{ Tabela 9: Satisfação com a aplicação de Jogos } \\
\hline Forma de aplicação & $N$ & Média & $\begin{array}{c}\text { Desvio } \\
\text { Padrão }\end{array}$ \\
\hline Disciplina específica & & & \\
Com software & 9 & 2,67 & 0,50 \\
Sem software & 10 & 2,50 & 0,53 \\
Outras disciplinas & & & \\
Com software & 10 & 2,80 & 0,42 \\
Sem software & 6 & 2,33 & 0,82 \\
\hline
\end{tabular}

Fonte: Elaborado pelos autores

No que diz respeito aos aspectos dificultadores e favorecedores à aplicação de jogos de empresas pelas IES, os coordenadores de curso foram solicitados a atribuir uma nota de 1 a 5, de acordo com o grau de concordância com as sete afirmações relativas aos aspectos dificultadores e também com as sete afirmações relativas aos aspectos favorecedores. Observa-se que a média atribuída é superior em todos os aspectos favorecedores em relação aos dificultadores, como apresentado na Tabela 10.

Mais uma vez, percebe-se a importância do custo de aquisição de jogos $(4,00)$ como o principal fator dificultador da utilização de jogos, seguido, com a mesma média, pela falta de professores capacitados. Esta ordem, dos dois principais aspectos, foi a mesma encontrada por Neves e Lopes (2008), referente às IES paulistas. 
Por outro lado, os aspectos com maior média entre os favorecedores à adoção dos jogos nos cursos de graduação das IES baianas são o estímulo ao trabalho em equipe $(4,81)$ seguido pela integração do conteúdo das diversas disciplinas e do estímulo ao pensamento sistêmico, esses últimos com a mesma média $(4,70)$.

Tabela 10: Aspectos facilitadores e dificultadores para implantação de jogos

\begin{tabular}{|c|c|c|c|}
\hline Aspectos & $N$ & Média & $\begin{array}{l}\text { Desvio } \\
\text { Padrão }\end{array}$ \\
\hline \multicolumn{4}{|l|}{ Dificultadores } \\
\hline O custo de aquisição dos jogos de empresas é elevado & 47 & 4,00 & 0,95 \\
\hline Faltam professores treinados para animar jogos de empresas & 47 & 4,00 & 1,12 \\
\hline Há dificuldade de acesso a jogos prontos & 47 & 3,30 & 1,27 \\
\hline A aplicação de jogos demanda muito tempo & 47 & 3,04 & 1,37 \\
\hline Os jogos não representam a realidade das empresas brasileiras & 47 & 2,89 & 1,07 \\
\hline Predomina infra-estrutura inadequada nas IES para uso de jogos & 47 & 2,87 & 1,48 \\
\hline Alguns coordenadores e IES não creditam importância aos jogos & 47 & 2,66 & 1,34 \\
\hline \multicolumn{4}{|l|}{ Favorecedores } \\
\hline Os jogos de empresas estimulam o trabalho em equipe & 47 & 4,81 & 0,45 \\
\hline Os jogos integram o conteúdo das diversas disciplinas do curso & 47 & 4,70 & 0,51 \\
\hline Os jogos estimulam o pensamento sistêmico & 47 & 4,70 & 0,55 \\
\hline Os jogos proporcionam importante aprendizagem cognitiva & 47 & 4,47 & 0,62 \\
\hline Os jogos são importantes na formação profissional & 47 & 4,45 & 0,77 \\
\hline O interesse de alunos por jogos de empresas é elevado & 47 & 4,11 & 1,00 \\
\hline É a ferramenta pedagógica que melhor aproxima teoria à prática & 47 & 4,02 & 1,07 \\
\hline
\end{tabular}

Fonte: Elaborado pelos autores

\section{Considerações Finais}

Há mais de 50 anos que os jogos de empresas são aplicados para o ensino de administração no mundo. No Brasil a adoção desta técnica foi tardia, com as primeiras experiências datando da década de 1970, sendo que só ocorreu de forma mais ampla a partir do ano 2000, como constatado por Bernard (2006), Arbex et al. (2006) e Neves e Lopes (2008). Observa-se que o Brasil está numa curva crescente da utilização de jogos nos cursos de graduação em administração e que os atuais índices deverão ser rapidamente superados, implicando uma larga adoção em todo o país nos próximos anos.

No caso específico da Bahia, mesmo o estado não tendo tradição, nem no desenvolvimento de software, nem na realização de estudos e pesquisas sobre o tema, constatou-se um índice de aplicação dos jogos igual a 44,7\%, o que se assemelha muito aos $41 \%$ das IES paranaenses (ARBEX et al., 2006) e aos 48,5\% das IES paulistas (NEVES; LOPES, 2008).

Dois pontos se destacaram como centrais a uma mais ampla utilização de jogos de empresas pelas IES baianas: o custo de aquisição e a oferta de simuladores eletrônicos. O REAd I Porto Alegre - Edição 72 - Nº 2 - maio/agosto 2012 - p. 317-338 
custo envolvido na oferta da técnica é o principal motivo para escolha de um determinado software, dentre aquelas IES que aplicam jogos através deste recurso. É também o principal motivo para a não utilização de software, dentre aquelas IES que aplicam jogos sem este recurso. E ainda é o principal motivo para a não aplicação de jogos de empresas.

Vicente (2001) classifica diversas formas distintas de aplicação de jogos de empresas (jogos de sala de aula, jogos de tabuleiro, jogos por e-mail, livro-jogo, dramatização e jogos de computador). Praticamente, todas as outras formas de aplicação de jogos, que não sejam através de software, têm custos provavelmente menores e ao menos potencialmente, supririam as necessidades das IES baianas. Entretanto, percebe-se que, provavelmente por conta da baixa capacitação dos professores e do fato de não haver uma disponibilização maior de jogos outros, a existência do software é determinante para a aplicação de jogos nas IES pesquisadas. A influência deste fator chega a superar a existência de uma disciplina (que demonstra um reconhecimento institucional da importância da técnica à formação do aluno) na aplicação de jogos.

Finalmente, é possível afirmar que há uma tendência de crescimento da utilização da técnica na Bahia, por se observar que os coordenadores das IES desse estado atribuem maior grau de concordância a todos os aspectos pesquisados como facilitadores do que àqueles pesquisados como dificultadores. Tem-se que esse conjunto de observações termina por justificar que se aprofunde a pesquisa sobre os jogos, sobre as formas de aplicá-los, suas características, consequiências e resultados.

\section{REFERÊNCIAS}

AAKER, D.A.; KUMAR, V. DAY, G.S. Pesquisa de Marketing. 2. ed. São Paulo: Atlas, 2004.

ARBEX, M. A.; CORRÊA, H. P.; MELO JR.; A., RIBAS, C. A.; LOPES, P. C. O uso de jogos de empresas em cursos de graduação em administração e seu valor pedagógico: um levantamento no estado do Paraná. In: XXX EnANPAD, 2006, Salvador. Anais... Salvador: ANPAD, 2006. 
BENNIS, W.; O’TOOLE, J. Como a escola de administração perdeu o rumo. Harvard Business Review, mai. 2005.

BEN-ZVI, T. The efficacy of business simulation games in creating decision support systems: an experimental investigation. Decision Support Systems 49, 61-69, 2010.

BEPPU, C. I. Simulação em forma de "jogo de empresas" aplicada ao ensino da Contabilidade. Dissertação (Mestrado em Administração) - Faculdade de Economia, Administração e Contabilidade, Universidade de São Paulo. São Paulo, 1984.

BERGGREN, C; SÖDERLUND, J. Management education for practicing managers: combining management education for practicing managers: combining. Journal of Management Education, 35 (3), 377-405, 2011.

BERNARD, R. Estrutura de utilização dos jogos de empresas nos cursos de graduação em administração e ciências contábeis do país e avaliações preliminares de uma disciplina baseada neste método. In: XVII ENANGRAD, 2006, São Luis. Anais... São Luis: ANGRAD, 2006.

BURGESS, T. F. The use of computerized management and business simulation in the United Kingdom. Simulation \& Gaming, 22 (2), 174-195, 1991.

CHANG, J. Use of business simulation games in Hong Kong. Simulation \& Gaming, 34 (3), 358-366, 2003.

FARIA, A. A survey of the use of business games in academia and business. Simulation \& Gaming, 18 (2), 207-224, 1987.

Business Simulation Games: Current Usage Levels - An Update. Simulation \& Gaming 29 (3), 295-308, sep. 1998.

FARIA, A.; HUTCHINSON, D.; WELLINGTON, W.; GOLD, S. Developments in business gaming : a review of the past 40 years. Simulation\& Gaming 40 (4), 464-487, 2009. 
GOLDSCHMIDT, P. C. Simulação e jogo de empresas. Revista de Administração de Empresas, Rio de Janeiro: FGV, v.17, n.3, p. 43-46, Mai-Jun, 1977.

GOOSEN, K.; MAURI, A.; RITCHIE, W.; WOLFE, J. Helping new game adopters: four perspectives. Developments in business simulation and experimental learning, ABSEL, v. 28, 80-91, 2001.

INEP (Instituto Nacional de Estudos e Pesquisas Educacionais Anísio Teixeira). Sinopses Estatísticas da Educação Superior - Graduação. Brasília, DF, 2006.

KEYS, J. B. Organizations Advancing Business Simulation and Experiental Learning. In: GENTRY, J. W. Guide to Business Gaming and Experiental Learning. London: Nichols/GP Publishing, 1990.

KEYS, B. e WOLFE, J. The role of management games and simulations in education and research: Yearly Review. Journal of Management, v. 16, n. 2, p. 307-336, 1990.

KOLB, D. Experimental Learning: experiance as the source of learning and development. New Jersey: Prentice Hall, 1984.

LACRUZ, A. J.; VILLELA, L. E. Percepção dos participantes de jogos de empresas quanto às condições facilitadoras para o aprendizado em programas de simulação empresarial: um estudo exploratório. In: XIX SEMEAD, 2006. São Paulo. Anais... São Paulo: USP, 2006.

LOPES, P. C. Formação de Administradores: uma abordagem estrutural e técnico didática. Tese (Doutorado em Engenharia de Produção) - Universidade Federal de Santa Catarina. Florianópolis, 2001.

MARTINELLI, D. P. A utilização de jogos de empresas no ensino de Administração. Dissertação (Mestrado em Administração) - Faculdade de Economia, Administração e Contabilidade, Universidade de São Paulo. São Paulo, 1987.

McKENNA, R. J. Business computerized simulation: the Australian experience. Simulation \& Gaming, 22 (1), 36-62, 1991. 
MENDES, J. B. A utilização de jogos de empresas no ensino da contabilidade - uma experiência no curso de ciências contábeis da Universidade Federal de Uberlândia. In: CONGRESSO BRASILEIRO DE CONTABILIDADE, 16, 2000. Goiânia. Anais... Goiânia, 2000 .

NAGAMATSU, F. A.; FEDICHINA, M. A. H.; GOZZI, S.; BOLDRIN, V. P. A aplicação do jogo de empresas no desenvolvimento gerencial: um estudo aplicado em cursos de graduação e de pós-graduação (nível lato sensu). In: XIX SEMEAD, 2006. São Paulo. Anais... São Paulo: USP, 2006.

NEVES, J. P.; LOPES, P. C. Jogos de empresas: um estudo da utilização em cursos de graduação em administração no estado de São Paulo. In. XXXII ENANPAD, 2008, Rio de Janeiro. Anais... Rio de Janeiro: ANPAD, 2008.

OLIVIER, M.; ROSAS, A. R. Jogos de empresas na graduação e no mestrado. In: VII SEMEAD, 2004. São Paulo. Anais... São Paulo: USP, 2004.

PAIXÃO, R. B.; BRUNI, A. L.; CARVALHO JR., C. V. O. Jogos de empresas na academia: aspectos conceituais e metodológicos de uma amostragem de publicações brasileiras entre 1998 e 2006. In: X SEMEAD, 2007. São Paulo. Anais... São Paulo: USP, 2007.

PEREIRA, M. Ensino-aprendizagem em um contexto dinâmico: o caso de planjamento de transportes. Tese (Doutorado em Engenharia) - Escola de Engenharia de São Carlos, Universidade de São Paulo. São Carlos, 2005.

POLZER, J.; GULATI, R.; KHURANA, R.; TUSHMAN, M. Crossing boundaries to increase relevance in organizational research. Journal of Management Inquiry, 18 (4), 280-286, 2009.

PROTIL, R. M.; FISCHER, H. Utilização de simuladores no ensino de ciências sociais aplicadas: um estudo na República Federal da Alemanha. In. XXVII ENANPAD, 2003, Atibaia. Anais... Atibaia: ANPAD, 2003.

ROCHA, L. A. Jogos de empresas: desenvolvimento de um modelo para aplicação no ensino de custos industriais. Dissertação (Mestrado em Engenharia da Produção). Florianópolis: Universidade Federal de Santa Catarina, 1997. 
ROSAS, A. R. ; SAUAIA, A. C. A. . Jogos de Empresas na Educação Superior no Brasil: Perspectivas para 2010. In: XXX EnANPAD, 2006, Salvador. Anais... Salvador: ANPAD, 2006.

SANTOS, R. V. Jogos de empresas aplicados ao processo de ensino e aprendizagem de contabilidade. Revista Contabilidade e Finanças, São Paulo, n.31, p. 78-95, jan./abr. 2003.

SAUAIA, A. C. A. Jogos de empresas: tecnologia e aplicação. Dissertação (Mestrado em Administração) - Faculdade de Economia, Administração e Contabilidade, Universidade de São Paulo. São Paulo, 1990.

A. Satisfação e aprendizagem em jogos de empresas: contribuições para a educação gerencial. Tese (Doutorado em Administração) - Faculdade de Economia, Administração e Contabilidade, Universidade de São Paulo. São Paulo, 1995.

Conhecimento versus desempenho das organizações: um estudo empírico com jogos de empresas. REAd - Edição 49 Vol. 12 No. 1, jan-fev 2006.

SHEIZI, C. F.; SANTOS, L. P. G. Jogos de Empresas: Um Estudo Exploratório sobre a Percepção e o Desempenho dos Alunos dos Cursos de Administração e Ciências Contábeis. In. XXVII ENANPAD, 2003, Atibaia. Anais... Atibaia: ANPAD, 2003.

TANABE, M. Jogos de empresas. Dissertação (Mestrado em Administração) - Faculdade de Economia, Administração e Contabilidade, Universidade de São Paulo. São Paulo, 1973.

THORELLI, H. Ecology of international business simulation games. Simulation \& Gaming, 32 (4), pp. 492-506, 2001.

TUSHMAN, M; O'REILlY, C; FENOLLOSA, A; KLEINBAUM, A; McGRATH, D.. Relevance and rigor: executive education as a lever in shaping practice and research. Academy of Management Learning \& Education, v. 6, n. 3, 345-362, 2007. 
VICENTE, P. Jogos de empresas: a fronteira do conhecimento em administração de negócios. São Paulo: Makron Books, 2001.

WILHELM, P. P. H.; LOPES, M. C. Uma nova perspectiva de aproveitamento e uso dos jogos de empresas. Revista de negócios, Blumenau: FURB, v.2, n.3, p. 43-55, Abr-Jun, 1997.

ZAJDSZNAJDER, Luciano. Experiências educacionais no ensino de Administração. In: Encontro Anual da Associação Nacional dos Programas de Pósgraduação em Administração, In. V ENANPAD, 1981. Anais... ANPAD, 1981. 